\title{
"Chronic Psychosis and Capgras Delusions associated with Megaloblastic Anaemia "(Report of a case)
}

\author{
TALIB S.H*, Bhattu S**, Sanober G****, Sainani Rahul ****, \\ Deshpande Shreyas \\ * Professor \& Head, ** Lecturer, **** Chief Resident \\ Department Of Medicine, Mahatma Gandhi Missions Medical College \& Hospital , Aurangabad.431003
}

\begin{abstract}
The combination of an anaemia and psychosis is noted more frequently then accounted by mere chance. The etiopathogenetic relationship of such anaemia to the development of such psychosis is complex and yet unclear. $B_{12}$ deficiency anaemia may coincide with psychosis developing before or after development of mental disorder. We report a case of young girl who developed psychosis 8 years back without features of anaemia and treated with anti psychotic therapies and ECT's before hospitalization with us as a case of severe megaloblastic anaemia and capgrass delusional psychosis. The clinicohematological, biochemical, histopathological, immunological and imaging studies undertaken in the case is reviewed and discussed. We humbly presume that the case presented with subtle $B_{12}$ deficiency and mental disorders with affection of temporal lobes either side could have resulted with neurotoxic damage of $B_{12}$ deficiencies and perhaps got deteriorated with ongoing several ECT's (60-70) which patient received over period of 8 years.
\end{abstract}

Key words : Megaloblastosis ,B12 deficiency, psychosis, capgras features.

\section{Introduction :}

Psychiatric Manifestations although are commonly associated with $\mathrm{B}_{12}$ deficiency anaemias, are uncommonly recognized. Early recognition and treatment of anaemia could prove fruitful in reducing the problems associated with neuropsychiatric manifestations of the disease. ECT's and / or neurotoxic damage to the brain because of occult and later manifest $\mathrm{B}_{12}$ deficiency is hypothesized to be responsible for patients chronic psychiatric, cappgrass - delusional status in the case .

\section{Case History :}

28 years old female having good parents staying in house with 1 brother and 3 sisters, patient being eldest of the sisters. She was intelligent and admitted to $1^{\text {st }} \mathrm{yr}$ Chemical Engineering, elder brother and 3 sisters are also well educated and persuaded engineering courses.

She was happy \& well mixed socially until she got A.T.K.T. in $1^{\text {st }}$ year of engineering . On the day of the result she was quite nervous and slept for long periods (reported to have not taken any pills) \& refused to take meals $\&$ became agitated. There was no family back ground of any psychiatric problem nor there was any history of drug consumption $\&$ any psychiatric therapy in past.Patient was seen for her irritability, despondency \& depression by local psychiatrist \& was put on antipsychotic therapy for nearly 1 year without fruitful outcome. Patient had series of psychiatric consultations from various places of Maharashtra state .

She had misidentification for parents and conveyed that "You are not my mother / you are not my father, they are dead, you are just imposter." This delusional misidentification persisted past 6-8 months .

In addition to antipsychotic therapy, she was provided with ECT (30-40) in period of 7 to 8 years. Further records of her past showed that she was non hypertensive, non diabetic and undergone nasal septal resection in June 2006. The record revealed that $\mathrm{Hb}$ done at that time was $11.5 \mathrm{gm} \%$. The psychotic problems further increased post resection. Patient never received any pills of vitamin during this period . Patient was vegetarian for the past 4 years. The patient's psychotic problem worsened. Irritability, irresistibility, Apathic attitude; Hallucination, paranoid features persisted. Agitating patient at times used to beat and break households glass articles \& refused to recognize parents as her own. ( Capgras delusions ).

Her remote memory was normal. Owing to her increasing drowsiness and extra pyramidal features, dehydration (patient on anti psychotic therapy chiefly serenace) she was hospitalized with us.

On examination she was noticed to have marked pallor $\mathrm{Hb} 1.5 \mathrm{gm} . \%$, blood pressure normal, No s/o failure, no any systemic problems except extra pyramidal rigidity and tremors. Signs of Dehydration were present and the patient was anorexic. She was irritable, hostile, non co operative, abusing and shouting to close relatives \& having hallucinatory behaviours and conceptual disorganization. MCV-104, RBC 2.42 mil, PLT $34000 \mathrm{SrB}_{12}$ level - 659 (N-211-911 pg/ml) Sr, ferritin 271.1(N-100-291 ng/ml), Peripheral smear revealed macrocytic normochromic red blood cells with hypersegmented polymorphs, bone marrow aspiration revealed 
abundant megaloblast (Fig-1) Intrinsic factor antibody NEGATIVE, antithyroid peroxidase antibodies 60.5 [Normal upto $60 \mathrm{dg} / \mathrm{ml}$ ) with normal thyroid function, Gastric fundal biopsy revealed Atrophic mucosa with fibrosis at sub mucosa. (Fig-2) MRI Brain done suggestive of temporal lobe atrophy. Serum gastrin level $18 \mathrm{u} / \mathrm{l}$ ( Normal), Blood homocystein level 6.41 [N3.36-20,44), LDH 1719 ( N313-618), Sr.folate level normal.

With these investigations patient was diagnosed to be case of MEGALOBLASTIC ANEMIA ( B12 deficiency ) WITH PSYCHOSIS . Patient received 03 PCV of blood along with 1000 ug of vit B12 alternate day for the first week, then once weekly and then fortnightly.

After 3 weeks, the haemoglobin improved from 1.5 to 10 gms.\% with improvement in her mental behaviour nevertheless she again started with paranoid ideas, hallucinatory behaviour, un- cooperativeness and self murmuring, however shouting and destructive nature was not observed. The psychiatrist recorded her BPAS (Behavioural psychiatric Rate Scoring) as 66 (Normal <20 -30), PANSS ( positive And Negative syndrome score ) as 116 (Normal 30). CGIS(Clinical global improvement score) as 5. She was found to have formal thought disorder alienation phenomena with affect blunted. At times she was very co-operative when confabulated with tender, loving care. The diagnosis was entertained as patient with psychosis , megaloblastic anemia with Capgras Disease.

\section{Discussion :}

The patient presented to us with severe anaemia of $1.5 \mathrm{gm} \%$ and chronic psychiatric manifestations having severe apathy, delusional states, irritability, irisability and non recognition of parents, shouts, disregards and misbehavior.

The presentation of the case has multifold problems as regards to the type of anaemia and psychosis whether occurring by chance or as a distinct pathogenetic mechanism of $\mathrm{B}_{12}$ deficiency. Folate deficiencies are rarely observed with neuropsychiatric problems ${ }^{2,3}$. We consider $B_{12}$ deficiency to be the cause of severely expressed maegaloblastic anaemia as there was megaloblastic picture on bone marrow \& fundal gastric biopsy revealed gastric atrophy and fibrotic changes \& the excellent response to the B12 therapy raising $\mathrm{Hb}$ from 1.5 to $10 \mathrm{gm} \%$.For pernicious anaemia recognition, parietal cell antibody although has sensitivity (90\%) but has poor specificity. Intrinsic factor antibody is very specific for pernicious anaemia with $100 \%$ specificity and $50-70 \%$ sensitivity. Gastric levels are noted elevated in $80-90 \%$ of cases of pernicious anaemia.The above findings were all within normal range.However we submit that these investigations were carried out after 8 days of treatment with B12.Methylmalonic acid was not done.Homocystine remained within normal range (as expected to be normal after 7 days of treatment).The normal ferritin levels in the case and absence of nutritional deficiency features rules out nutritional cause of B12 deficiency.

The differences in the individual conception of psychiatric changes are wide $\&$ includes features like apathy, inco-operativeness, irritability, apprehensive neurosis, paranoid states, delusional confessional states, unpleasant hallucinations and prosopagnosia like features ${ }^{1,4,5,6 .}$. Capgras syndrome is a disorder in which a person holds a delusion that a friend, spouse, parents \& other close family members have been replaced by an identical looking imposters $\&$ the beliefs involve the misidentification of people, place, objects. The patient easily recognized other family members \& would misidentify her parents only, as noted in our case ${ }^{5} \cdot B_{12}$ belongs to the family of cobalamines and it serves as a cofactor for 2 important reactions in humans. As methylcobalamin is a co-factor of methionine synthetase enzyme for conversion of homocysteine to methionine and secondly as Adenosyl cobalamine for conversion of methyl malonyl COA to succinyl coenzyme A. The deficiency is often noted in vegans, strict vegetarians as was with our patient. The liver is known to contain 2000-5000ug as stored $\mathrm{B}_{12}$. Deficiency usually takes place more than 3 years after the absorption ceases. The defect in methylation process is considered vital for the neuropsychiatric manifestations. Homocystine and MMA are useful metabolic markers for $\mathrm{B}_{12}$ deficiency and it is therefore desirable to check them early before instituting the therapy ${ }^{3}$

Temporal lobes automatism is maintained by monoamino transmitter synthesis which is derived from tetrahydrobiopterynes in the presence of $B_{12}$. Lack of $B_{12}$ leads to poor neurotransmitter synthesis and causes neuronal destruction with increased levels of methyltetrahydrofolate (MTHF) levels. ${ }^{7}$

Temoral lobe atrophy is described in many cases who received regular ects for long periods ${ }^{8}$. In the present case, MRI revealed bilateral temporal atrophy ,the neuronal damage could be secondary to megaloblastic $\mathrm{B}_{12}$ deficiency and perhaps series of ECT's which patient received in past 8 years. Serum $\mathrm{B}_{12}$ levels may remain very low or normal in established $\mathrm{B}_{12}$ deficiencies. The neuronal $\mathrm{B}_{12}$ levels invariably falls earlier than the serum ${ }^{9}$

The patient's condition considerably improved on therapy, however having waxing waning features of delusional status. Non recognition of only parents persisted, the destructive violent features however are not observed after 1 year of follow up.

Conclusion - $\mathrm{B}_{12}$ deficiency treatment may revert the psychosis completely when treated early i.e within 6 months to 1 year of onset of illness. The total recovery may not be possible when treated inadequately at early 
stage. Treatment when instituted late in the course of illness may not show totally a fruitful outcome as found in the present case.A caution for regular use of ECTs to such category of patients is warranted in view of adverse effects of ECTs on temporal lobe.

\section{Legends to figures :}

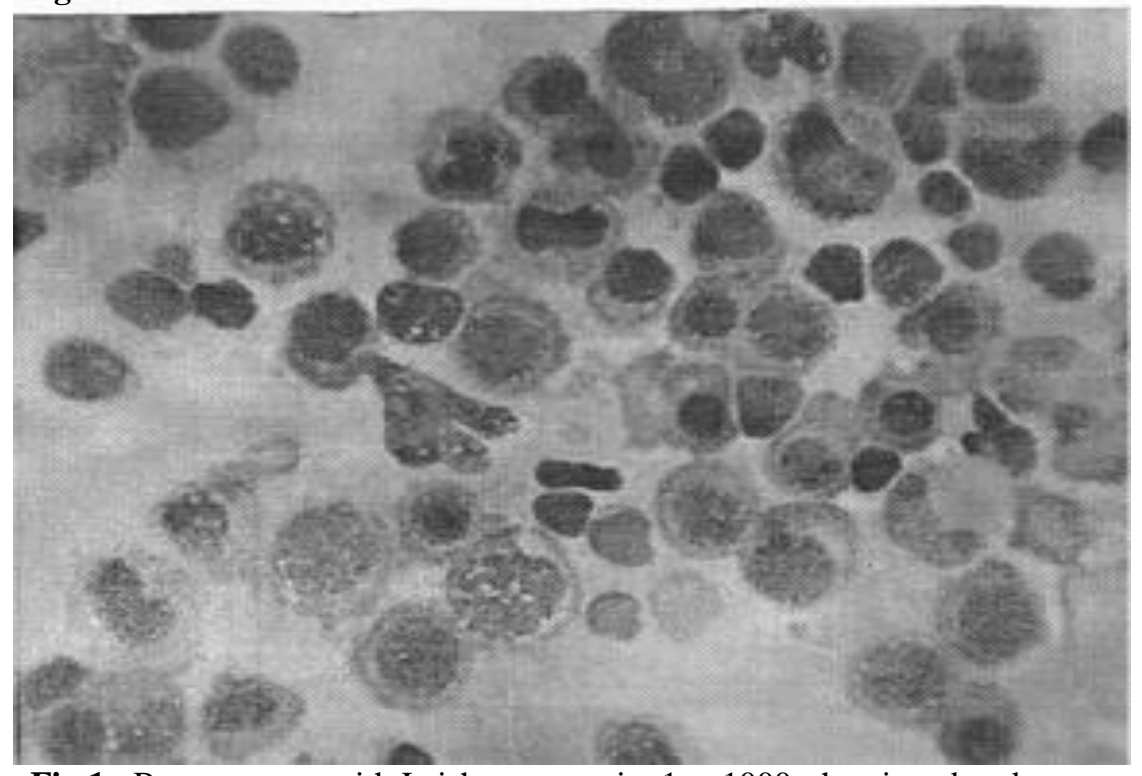

Fig 1 : Bone marrow with Leishmanns stain 1 x 1000 showing abundant megaloblasts.

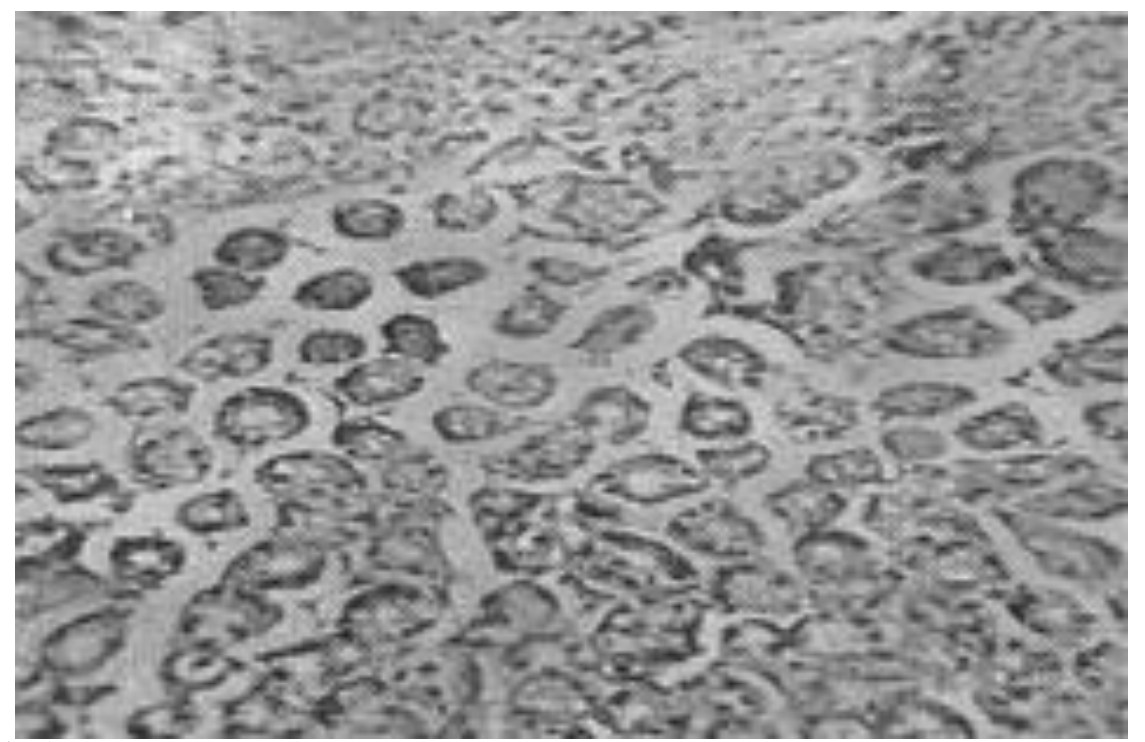

Fig 2 : Fundal gastric biopsy with $\mathrm{H} \& \mathrm{E}$ stains ) revealing gastric atrophy \& fibrosis.

\section{References :}

[1]. Durand C, Mary S, Brazo P, Dollfus S .Psychiatric Manifestations of Vitamin B B Deficiency . Encephale 2003; 29 (6) : 560-565

[2]. Herr KD , Norris ER, Frankel BL, Acute Psychosis in a patient with Vit $\mathrm{B}_{12}$ deficiency \& coincident cevical stenosis. Psychosomatics 2002; 43:234-236

[3]. Allen RH Lindenbaum J \& Stabler S P .High prevalence of Cobalamine deficiency in the elderly. Transactions of the American Clinical \& Climatological Association1995; 107:37-47.

[4]. Payinda G, Hansen T. Vitamin B12 Deficiency manifested as psychosis without anaemia, American Journal of Psychiatry 2000; $157: 4,660-661$

[5]. http.// en.wikipedia.org/wiki/Capgras delusion Diseases DB 3260622 Jun $2011,1-5$

[6]. Parfitt DN . Psychosis associated with Pernicious anaemia with a report of 2 cases. Journal Of Neurology \& Psychopathology 1934:15 $; 12-19$.

[7]. Rajkumar A. Jebaraj P. Chronic Psychosis associated with Vit B12 deficiency JAPI 2008;56:115-116.

[8]. Friedberg JM. Recurrent ECT Therapy leads to temporal lobe damage. American Journal of Psychiatry 1977;134:9,1010-1013.

[9]. Aaron S, Kumar S, Vijayan J, Jacob J, Alexander M, Gnanamuthu C. Clinical and laboratory features and response to treatment in patients presenting with vitamin B12 deficiency related neurological syndromes . Neurol India 2005;53:55-58. 